\title{
Serum metallothionein - a potential oncomarker?
}

\author{
Horakova Z, Starek I \\ Clinic of Otorhinolaryngology and Neck Surgery, Faculty of Medicine and Dentistry, Palacky University \\ and University Hospital Olomouc, Olomouc, Czech Republic. zuzana.horakova@fnol.cz
}

\begin{abstract}
INTRODUCTION: Metallothionein's (MT) overexpression has been demonstrated immunohistochemically in neoplastic cells of many tumour types. Its elevation above the physiological level has been confirmed in circulation of their hosts. The results of studies dealing with the topic have been summarized to verify if this marker can be applied in the current oncologic practise.

METHODS: The Pubmed and Google Scholar medical databases were reviewed for full-text articles focused on MT blood (plasma / serum) levels in patients with malignant tumours.

RESULTS: In our review, after a precise selection, we included 8 prospective randomized trials encompassing 561 blood samples taken from patients with a large histopathological spectrum of malignancies. In general, significant differences in blood MT levels between oncological patients and healthy subjects were confirmed. No particular value of the MT level has been demonstrated to be unequivocally predictive of oncologic disease CONCLUSION: The results of our review suggest that although the elevation of MT in blood serum in patients with solid malignancy can be regarded as a promising tumour marker, the recommendations of its applicability in clinical practice require to be derived from further research on extended cohorts of patients (Tab. 1, Fig. 1, Ref. 49). Text in PDF www.elis.sk

KEY WORDS: metallothionein, tumour marker, solid tumour.
\end{abstract}

\section{Introduction}

A tumour marker is defined as a parameter capable of indicating the presence or progression of a tumour with sufficient sensitivity and specificity. Clinical applications of oncomarkers can be divided into screening, diagnosis, prognosis, prediction of the therapeutic response and monitoring of tumour's biological activity (1).

For some malignancies, nonspecific markers (SCCAg, CEA, CYFRA 21-1 etc) are being used in the above-mentioned applications, while the use of specific markers (e.g., PSA) is rare. For other tumours, including the head and neck squamous cell carcinoma (SCC), the former show small effectiveness and the latter have not been identified so far (2, 3).

Metallothionein (MT) belongs to the superfamily of metalbinding proteins present in the cells of all living organisms (4). In low concentrations, this protein can be detected in some body fluids, e.g., in plasma, bile, urine, and saliva (5).

MT is involved in many metabolic pathways (4). Its synthesis and consequently, its plasmatic concentrations are controlled by various factors, primarily by metal ions, glucocorticoids, acute

Clinic of Otorhinolaryngology and Neck Surgery, Faculty of Medicine and Dentistry, Palacky University and University Hospital Olomouc, Olomouc, Czech Republic

Address for correspondence: Z. Horáková, KORLHK, FN Olomouc, IP Pavlova 6, CZ-779 00 Olomouc, Czech Republic.

Phone: +420776240518 phase proteins, inflammatory cytokines and stress mediators $(6,7,8)$.

It is generally accepted that MT plays a very important role in heavy metal detoxification, pooling and regulation of copper and zinc ions, with the latter being essential in regulating the activity of DNA repair enzymes. Moreover, MT acts as a free radical scavenger protecting cell structures against acute inflammation and associated oxidative stress. $(9,10,11,12)$

The serum MT concentration in healthy individuals may be influenced by many physiological factors (age, sex, food supplement intake, physical exercises), as well as environmental and geographical circumstances $(13,14)$.

Moreover, increased blood levels of this protein have been demonstrated under various pathologic conditions (e.g., inflammation, injury, cardiovascular or endocrine disease, hepatopathy) (15).

Until now, four MT isoforms expressed in human cells have been identified, namely MT1 (subtypes A, B, E, F, G, H, L, M, X), MT2 (subtypes A, B), MT3, and MT4. Particular isoforms which are similar to each other in their physical and chemical characteristics are produced in different proportions in various tissues and herein degraded at different ratios. MT1 and MT2 are ubiquitous whereas the minorities MT3 and MT4 were detected in human brain and stratified squamous epithelium of the skin, oesophagus, and tongue, respectively (Miles). MT3 and MT4 are present constantly and independently from signals of induction by pathologic alterations, as opposed to MT1 and MT2, the expression of which is stimulated by many various stimuli (vide supra) (16). 


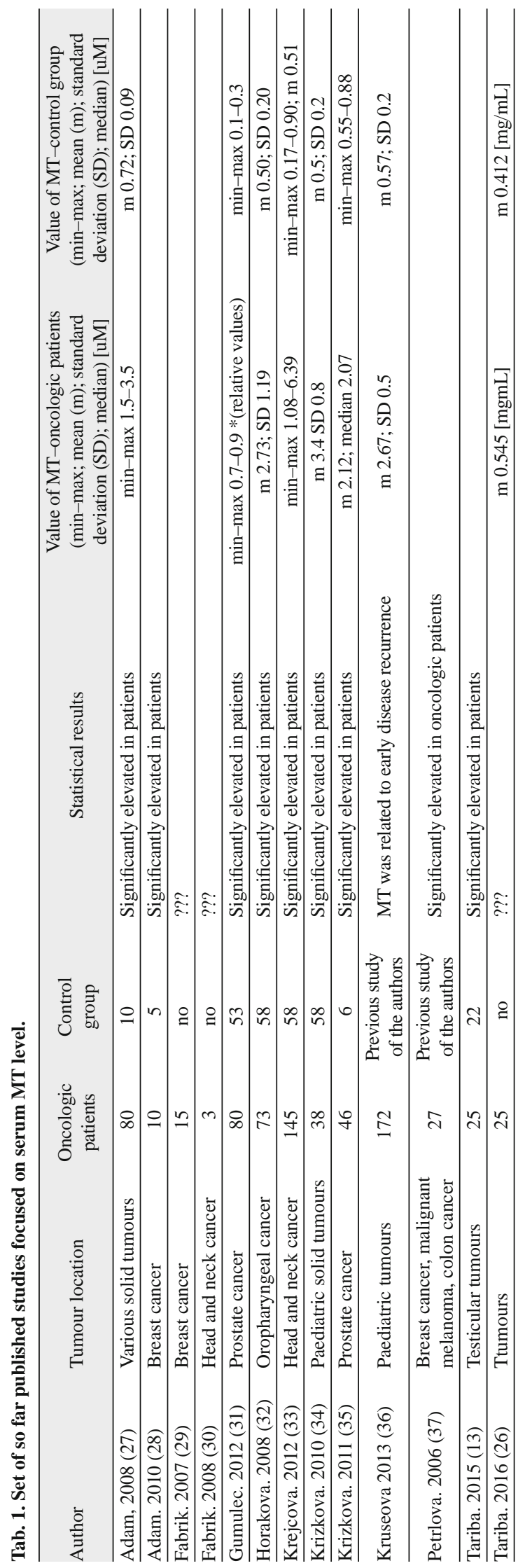

MT is suggested to be involved also in cancerogenesis ( $\mathrm{Si}$ ). However, relevant specific mechanisms have not been elucidated as yet (17). Woo demonstrated MT activity mainly in the early phase of that process (17); Pastuszewski and Ioachim assumed that MT overexpression is associated with oncogenous dedifferentiation of cells elicited by reexpression of primarily suppressed oncoproteins $(18,19)$.

In tumorous tissues, MT was found to be overexpressed not only in neoplastic cells but also in associated stromal fibroblasts, which supports the theory of remodelling of the microenvironment by tumoral cells, while thereby promoting their local and nodal metastatic spread (20).

Immunohistochemical MT expression in tumorous tissues was tested in numerous studies. In 2014, Gumulec published a large meta-analysis of 77 studies on MT expression comprising 4,631 various solid malignancies. As opposed to the correspondent adjacent healthy tissues, the increase in MT expression was found in all but hepatic tumours. The differences were significantly increased, primarily in head and neck, mammary, ovarian, uterine and prostate carcinomas (21). Moreover, some recent studies have demonstrated significant correlations of MT overexpression with tumour grade (22), metastatic spread (23), prognosis and radiochemo-resistance in breast cancer (24).

Previous studies tested the expression of complex MT in neoplastic tissues without having analysed its particular isoforms (25). These were identified in recent trials, while demonstrating the prevalence of MT1, lower prevalence of MT2 and only sporadic expression of MT3 and MT4.

Thus, a question can be raised as to whether the serum MT concentration could reflect the presence and biological activity of tumours and consequently act as an efficient tumour marker. Up to now, only a limited number of papers dealing with that topic has been published. We therefore review their conclusions to delineate further conceivable investigation in this perhaps promising field of oncology.

\section{Materials and methods}

The search was performed using the PubMed (Medline 1968 to November 2018) and Google Scholar, and subsequently, in bibliographies of identified references. Following keywords were used: metallothionein, serum or plasma or blood circulation, tumour or neoplasm or malignancy or cancer or carcinoma. The language was not a restricting selection criterion. Full-text articles were included only. If duplicated data were found in subsequent studies published by the same author(s), only the more comprehensive were taken into consideration. Following information was extracted from the studies: (1) blood MT level in patients with malignant tumours and set of healthy controls, (2) tumour location and histology.

Altogether, 13 studies were found to be analysing serum MT concentrations in oncologic patients. Four studies were subsequently excluded as they dealt mainly with the methodology of MT blood analysis without providing sufficient relevant clinical and/or histopathological data. Identical sets of patients were reported in two consecutive papers by Tariba $(13,26)$ and therefore, 


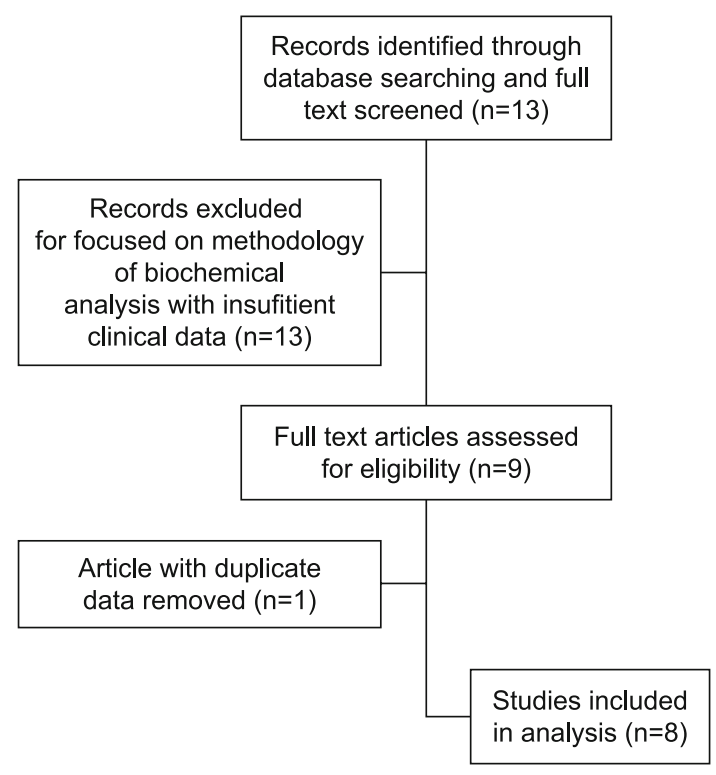

Fig. 1. Flow diagram of literature search and study selection process.

only those referred in the latter were included (26). Eventually, eight randomized prospective studies were involved in our analysis. The process of literature reviewing is illustrated in a flow diagram (Fig. 1) and details of the finally included studies are summarized in Table 1.

\section{Results}

The total of patients enclosed was 579, of whom 561 were treated for histologically verified solid malignancies (351 adults and 210 children) and 18 for leukemia. In all patients, blood sampling preceded the therapy (Tab. 1). With 218 cases, head and neck cancer (HNC) was the prevailing entity $(30,32,33)$, followed by 126 cases of prostate carcinoma $(31,35)$ and 25 cases of testicular carcinoma (13). Remaining cases were of other histological types and locations (28, 35, 36, 37) (Tab. 1).

All 8 analysed studies compared serum MT concentrations in oncological cases to those of control groups comprised of nononcologic healthy volunteers. The results including the values of MT concentration and statistical significance between the two groups are summarized in Table 1 . The results of most of the cited studies confirmed a significant elevation of MT serum level in oncological patients.

In the majority of trials (Tab. 1), solely means and standard deviations of the MT concentration were reported: the former varied from 2.12 to $3.4 \mathrm{uM}$ in oncologic and from 0.5 to $0.72 \mathrm{uM}$ in control groups.

In other two $(28,33)$ analyses comprising a total of 225 oncologic patients, only value ranges were available: the MT levels in oncologic and control groups varied greatly from 1.08 to $6.39 \mathrm{uM}$ and from 0.17 to $0.9 \mathrm{uM}$, respectively.

\section{Discussion}

Many trials dealt with immunohistochemical MT expression in samples taken from a broad histopathologic spectrum of neoplasms. Their results were summarized by Gumulec (21), who confirmed MT expression in tumours to be higher than in adjacent healthy tumour-free tissues (38). In their recent comprehensive literature review, Si pointed out the association between immunohistochemical MT overexpression in some tumours and their prognosis, chemo-/ radio- resistance, stage and grade (38).

On the contrary, only a few studies dealt with blood MT levels in oncologic patients. All the eight analysed trials demonstrated that serum MT concentrations were significantly higher in oncologic patients than those in healthy controls (Tab. 1), including the two largest ones analysing 218 head and neck cancers (32, 33).

Six studies (Tab. 1) reported MT level means and medians with low standard deviations indicating a relative homogeneity of results. In other two studies dealing with various solid malignancies (28) and head and neck carcinomas (33), large ranges of individual blood MT levels were reported. However, neither means, nor medians and standard deviations were available, making farther comments impossible.

Our review demonstrated that in oncologic patients, the ranges of blood MT concentration values were wider than in healthy individuals. We suggest that this may reflect the specificity of particular oncopathological groups, as well as induced biologic response and individual variability of the hosts. Our opinion is further supported by the results of two other studies, in which the variabilities of serum MT concentrations in control groups were generally low, while not exceeding $1.0 \mathrm{uM}$, (32, 33).

Considering the great variability of significantly elevated blood MT concentrations in oncologic patients and homogeneity of generally low MT levels in healthy probands, we suggest that physiological factors (such as sex and age) and environmental conditions have no substantial impact on MT levels in the former group. Our opinion seems to be supported by a study (31) analysing the impact of hypertension, hyperlipidaemia, ischaemic heart disease and duodenal ulcer on serum MT levels in patients diagnosed with solid malignancies. The comorbidities had no effect on concentrations of this protein. The result concerts with another trial (36) confirming no association between the serum MT concentration and inflammation blood markers in oncologic patients (36).

A relatively great interindividual variability of the MT level in oncologic cases may be explained by histopathologic diversity of tested tumours and by individual biological response of the host organism.

The relations between clinical and histopathological characteristics of tumours, tissue MT overexpression and blood MT concentrations were tested in the studies as follows.

Krizkova found that in detection of a prostate carcinoma, the combination of MT and PSA was superior to the latter marker alone; the MT level showed high specificity and markedly reduced the high false positivity of PSA (35). Surprisingly, Gumulec found an inverse relation between blood MT level, Gleason histopathological score and prognosis in patients with malignancy 
(31). Tariba confirmed an association between MT levels and clinical stages of testicular cancer (26). Krejcova tested blood MT levels in patients with squamous cell head and neck carcinomas. The average values for particular tumour locations (larynx, oropharynx, hypopharynx, oral and nasal cavity) varied and showed a slight tendency to increase with more advanced T stage and presence of nodal metastases. The lowest MT serum concentration in oncologic group was $1.09 \mathrm{uM}$, while in healthy individuals, the level did not exceed $0.9 \mathrm{uM}$. The author consequently suggested the 1-uM level as the cut-off value with that level showing $100 \%$ sensitivity and specificity (33). Kruseova analyzed MT blood concentrations in 865 samples of 172 paediatric patients with solid malignancies treated with chemotherapy. Cases with persistent or early recurrent disease showed that their MT concentrations at the beginning and during the treatment were significantly lower than in those achieving long-term remissions. The results suggested that in the latter group, the therapy applied was more efficient due to escalated oxidative stress and cell damage. In patients in long-term remission (follow-up ranging from 18 to 39 months), a decrease in MT levels lasted for 2 months after the treatment had been finished (36).

Other studies failed to find any association between the blood MT concentrations and tumour characteristics. No relation of MT with the clinical stage or histological grade was found in oropharyngeal SCC (32) and solid paediatric malignancies (36). Moreover, MT blood concentration showed no predictiveness for the response to chemotherapy in prostatic cancer (35).

Krizkova performed a large literature review focused on tissue expression of particular MT isoforms, their ratio and prognostic significance in various types of malignancies. She concluded that the majority of them show upregulation of MT1, MT2 and rarely also MT3. Expression patterns of particular MT isoforms varied in various types of cancers and had no prognostic relevance (39). The only two exceptions were studies on breast cancer in which increased tissue MT3 portended a poor outcome $(40,41)$.

So far, no study identifying particular blood MT isoforms in oncologic patients has been performed. Consequently, it is not clear whether they would have diagnostic, predictive o prognostic relevance. As suggested by some authors, an analysis of the oncologically relevant MT isoforms might also contribute to better understanding of their role in cancerogenesis $(17,18,19)$.

For quantitative analysis of blood MT levels, various methods can be used. In all the reviewed trials, an electrochemical voltametric detection (Brdicka reaction) of complex MT was applied (13, $28,29,30,37,39,42)$. This very sensitive method of identifying MT on zeptomol level is based on differential pulse voltametric analysis of the $\mathrm{SH}$ group, which is the fraction dominating its structure. The MT concentration values are subsequently calculated from the specific catalytic peak of voltammogram. The capability of voltametric reaction to discriminate particular MT isoforms is intensively tested now $(28,44)$.

As for the blood MT analysis, other authors used radioimmunoassay (RIA) $(45,46)$ or enzyme-linked immunoassay (ELISA) $(47,48,49)$ which showed the capacity to detect the human serum MT, the levels of which reach the order of uM. Unfortunately, the methods are largely limited by current absence of standardized specific antibodies necessary for MT detection.

As none of these analytical methods are routinely used, the precise assessment of the clinical relevance of MT serum concentrations has not been performed yet.

\section{Conclusions}

The systematic literature review comprised 8 up to now published studies in which relevant clinical data were available. It revealed that in comparison to healthy probands, means and ranges of blood MT concentrations in patients diagnosed with a broad histopathologic spectrum of solid malignancies are significantly higher with a suggested cut-off value of concentration of $1 \mathrm{uM}$ for oncologic patients.

However, the prognostic and predictive relevance of blood MT levels in clinical oncology remains unclear. Consequently, further research is necessary. It should be performed on large cohorts of patients stratified into specific oncological groups, with sufficient follow-ups. For the purpose of blood MT analysis, reliable and standardized laboratory methods must be applied. As the Brdicka reaction is not available in standard clinical labs, the alternative in form of ELISA and RIA should be preferred. All these methods are capable of detecting particular ontologically relevant MT isoforms, the analysis of which seems to be essential for further research in this field of clinical oncology.

\section{References}

1. Hajduch M, Jarosová M, Trojanec R et al. Cytogenetics and molecular biological markers in oncology and hematooncology. Klin Onkol 2004; 17 (Suppl 1): 51-56.

2. Yasumatsu R, Nakano T, Hashimoto K, Wakasaki T. The clinical value of serum squamous cell carcinoma antigens 1 and 2 in head and neck squamous cell carcinoma. Auris Nasus Larynx 2019; 46 (1): 135-140.

3. Liu L, Xie W, Xue P, Wei Z, Liang X et al. Diagnostic accuracy and prognostic applications of CYFRA 21-1 in head and neck cancer: A systematic review and meta-analysis. PLoS ONE 2019; 14(5): e0216561.

4. Vallee B. The function of metallothionein. Neurochem Int 1995; 27: 23-33.

5. Akintola DF, Sampson B, Fleck A. Development of an enzyme-linked immunosorbent assay for human metallothionein-1 in plasma and urine. J Lab Clin Med 1995; 126 (2): 119-27.

6. Rahman MT, Haque N, Abu Kasim NH, De Ley M. Origin, function, and fate of metallothionein in human blood. Rev Physiol Biochem Pharmacol. 2017; 173: 41-62.

7. Haq F, Mahoney M, Koropatnick J. Signaling events for metallothionein induction. Mutation research 2005; 33: 211-226.

8. Li Y. Zinc sensing mechanism involves linker peptides between the zinc fingers. Mol cell biol 2006; 26: 5580-5587.

9. Penkowa M, Keller P, Keller C et al. Exercise induced metallothionein expression in human sceletal muscle fibres. Exp physiol 2004; 90: 477-486.

10. Viarengo A, Burlando B, Ceratto N, Panfoli I. Antioxidant role of metallothioneins a comparative overview. Cellular and molec biology 2000; 46: 407-417.

11. Andrews G. Regulation of metallothionein gene expression by oxidative stress and metal ions. Biochem pharmac 2000; 59: 95-104.

12. Sato M, Kondoh M. Recent studies on metallothionein protection against toxicity of heaby metals and oxygen free radicals. J exp med 2002; 196: 9-22. 
13. Tariba B, Zivkovic T, Krasnic N, Filipovic V, Marijic V et al. A serum metallothionein in patients with testicular cancer. Cancer Chemother Pharmacol 2015; 75 (4): 813-820.

14. Nakazato K, Tomioka S, Nakajima K et al. Determination of the serum metallothionein (MT) $1 / 2$ concentration in patients with Wilson's disease and Menkes disease. J Trace Elem Med Biol 2014; 28 (4): 441-447. DOI: 10.1016/j.jtemb.2014.07.013.

15. Rodrigo MAM, Jimenez JAM, Haddad Y et al. Metallothionein isoforms as double agents-their roles in carcinogenesis, cancer progression and chemoresistance. Drug Resistance Updates Available online 4 March 2020, 100691 In Press.

16. Mehus AA, Muhonen WW, Garrett H, Somji S et al. Quantitation of human metallothionein isoforms: A family of small, highly conserved, cysteine-rich proteins. Mol Cell Proteomics 2014; 13 (4): 1020-1033.

17. Woo E, Kondo Y, Watkins S, Hoyt D, Lazo J. Nucleophilic distribution of metallothionein in human tumor cells. Experimental cell research 1996; 224: 365-371.

18. Pastuszewski W, Dziegiel P, Krecicki T, Podhorska-Okolow M, Ciesielska U et al. Prognostic significance of metallothionein, p53 protein and Ki-67 antigen expression in laryngeal cancer. Anticancer Res 2007; 27 (1A): 335-342.

19. Ioachim E, Assimakupoulos D, Peschos D, Zissi A, Skevas A. et al. Immunohistochemical expression of metallothionein in benign premalignant and malignant epithelium of the larynx. Pathol Res Pract 1999; 195: 809-814.

20. Dutsch-Wicherek M, Lazar A, Tomaszewska R et al. Analysis of metallothionein and vimentin immunoreactivity in pharyngeal squamous cell carcinoma and its microenvironment. Cell Tissue Res 2013; 352 (2): 341-349.

21. Gumulec J, Raudenska M, Adam V. Metallothionein-Immunohistochemical cancer biomarker: a meta-analysis. Plos One 2013; 9 (1): e85346.

22. Sampaio FA, Martins LM, Dourado et al. A case-control study of Metallothionein-1 expression in breast cancer and breast fibroadenoma. Sci Rep 2019; 9:7407. https://doi.org/10.1038/s41598-019-43565-0.

23. Szelachowska J, Dziegiel P, Jelen Drzeszewsaka J et al. Correlation of metallothionein expression with clinical progression of cancer in the oral cavity. Anticancer Res 2009; 29: 589-595.

24. Yap X, Tan HY, Huang J et al. Over expression of metallothionein predicts chemoresistance in breast cancer. J Pathol 2009; 21: 563-570.

25. Thirumoorthy N, Sunder AS, Manisenthil Kumar KT, Senthil Kumar $\mathbf{M}$ et al. A review of metallothionein isoforms and their role in pathophysiology. World J Surg Oncol 2011; 9: 54.

26. Tariba B, Zivkovic T, Filipovic V, Erk M, Gamulin M et al. Does the serum metallothionein level reflect the stage of testicular germ cell tumor. Arch Med Res 2016; 47 (3): 232-235.

27. Adam V, Blastik O, Krizkova S, Lubal J, Kukacka J,Prucha R et al. Application of the Brdicka reaction in determination of metallothionein in patients with tumours. Chem Listy 2008; 102 (1): 51-58.

28. Adam V, Petrlova J, Wang J, Eckschlager T, Trnkova L et al. Zeptomole electrochemical detection of metallothioneins. PLoS ONE 2010; 5 (7): e11441.

29. Fabrik I, Adam V, Krizkova S, Kukacka J, Prusa R et al. Level of heat stable thiols in patients with a malignant tumor. Klin Onkol 2007; 20 (6): 384-389.

30. Fabrik I, Krizkova S, Huska D, Adam V, Hubalek J et al. Employment of electrochemical techniques for metallothionein determination in tumor cell lines and patients with a tumor disease. Electroanalysis 2008; 20: 1521-1532.

31. Gumulec J, Masarik M, Krizkova S et al. Evaluation of alpha-methylacyl-CoA racemase, metallothionein and prostate specific antigen as prostate cancer prognostic markers. Neoplasma 2012; 59 (2): 191-201.
32. Horakova Z, Tothova E, Salzman R, Binkova H, Fabrik I et al. Importace of elevation of metallothionein blood levels in patients with head and neck tumors. Otorinolaryng Foniatr 2008; 57: 90-97.

33. Krejcova L, Fabrik I, Hynek D et al. Metallothionein electrochemically determined using Brdicka reaction as a promising blood marker of head and neck malignant tumours. R Int J Electrochem Sci 2012; 7: 1767-1784.

34. Krizkova S, Masarik M, Majzlik P, Kukacka J, Kruseova J et al. Serum metallothionein in newly diagnosed patients with childhood solid tumours. Acta Biochim Pol 2010; 57: 561-566.

35. Krizkova S, Ryvolova M, Gumulec J, Masarik M, Adam V et al. Electrophoretic fingerprint metallothionein analysis as a potential prostate cancer biomarker. Electrophoresis 2011; 32: 1952-1961.

36. Kruseova J, Hynek D, Adam V, Kizek R, Prusa R et al. Serum metallothioneins in childhood tumours-a potential prognostic marker. Int J Mol Sci 2013; 14: 12170-12185.

37. Petrlova J, Blastik O, Prusa R et al. Determination of metallothionein content in patients with breast cancer, colon cancer and malignant melanoma. Klin Onkol 2006; 19 (2): 138-142.

38. Si M, Lang J. The roles of metallothioneins in carcinogenesis. J Hematol Oncol 2018; 11: 107.

39. Krizkova S, Fabrik I, Adam V et al. Utilizing of adsorptive transfer stripping technique Brdicka reaction for determination of metallothioneins level in melanoma cells, blood serum and tissues. Sensors 2008; 8: 3106-3122.

40. Sens MA, Somji S, Garrett SH, Sens DA. Metallothionein isoform 3 (MT-3) overexpression is associated with breast cancers having a poor prognosis. Am J Pathol 2001; 159: 21-26.

41. Somji S, Garrett SH, Zhou XD, Zheng Y, Sens DA et al. Absence of metallothionein 3 expression in breast cancer is a rare, but favorable marker of outcome that is under epigenetic control. Toxicol Environ Chem 2010; 92 (9): 1673-1695. DOI:10.1080/02772241003711274.

42. Adam V, Beklova M, Pikula J, Hubalek J, Trnkova L et al. Shapes of differential pulse voltammograms and level of metallothionein at different animal species. Sensors 2007; 7: 2419-2429.

43. Adam V, Petrlova J, Wang J, Eckschlager T et al. Zeptomole electrochemical detection of metallothioneins. PLoS One 2010; 5 (7): e11441.

44. Merlos Rodrigo MA, Molina-López J, Jimenez Jimenez AM et al. The application of curve fitting on the voltammograms of various isoforms of metallothioneins- metal complexes. Internat J Mol Sci 2017; 18 (3). DOI: 10.3390/ijms18030610.

45. Nakajima K, Suzuki K, Otaki N, Kimura M. Epitope mapping of metallothionein antibodies. Methods Enzymol 1991; 205: 174-189.

46. Mulder TPJ, Janssens AR, Verspaget HW, Lamers CBHW. Development of a radioimmunoassay for human metallothionein. J Immunol Methods 1990; 130: 157-161.

47. Nagamine T, Nakajima K.Nagamine T et al. Development of a high sensitivity ELISA for the assay of metallothionein. Curr Pharm Biotechnol 2013; 14 (4): 427-431.

48. Nakazato K, Tomioka S, Nakajima K, Saito $\mathrm{H}$ et al. Determination of the serum metallothionein (MT)1/2 concentration in patients with Wilson's disease and Menkes disease. J Trace Elem Med Biol 2014; 28 (4): 441-447.

49. Jia Q, Dahms HU, Wang L. Detection of metallothionein proteins by enzyme-linked immunosorbent assay (ELISA). Curr Pharm Biotechnol 2020; 21 (7): 544-554.

Received January 26, 2021. Accepted February 13, 2021. 